This item was submitted to Loughborough's Research Repository by the author.

Items in Figshare are protected by copyright, with all rights reserved, unless otherwise indicated.

\title{
Dual antenna selection in self-backhauling multiple small cell networks
}

PLEASE CITE THE PUBLISHED VERSION

http://dx.doi.org/10.1109/LCOMM.2016.2570226

PUBLISHER

(c) IEEE

VERSION

NA (Not Applicable or Unknown)

LICENCE

CC BY-NC-ND 4.0

REPOSITORY RECORD

Chen, Gaojie, Yu Gong, Pei Xiao, and Rahim Tafazolli. 2019. "Dual Antenna Selection in Self-backhauling Multiple Small Cell Networks". figshare. https://hdl.handle.net/2134/23646. 


\title{
Dual Antenna Selection in Self-Backhauling Multiple Small Cell Networks
}

\author{
Gaojie Chen, Yu Gong Member, IEEE, Pei Xiao and Rahim Tafazolli, Senior Member, IEEE
}

\begin{abstract}
This paper investigates self-backhauling with dual antenna selection at multiple small cell base stations. Both half and full duplex transmissions at the small cell base station are considered. Depending on instantaneous channel conditions, the full duplex transmission can have higher throughput than the half duplex transmission, but it is not always the case. Closed-form expressions of the average throughput are obtained, and validated by simulation results. In all cases, the dual receive and transmit antenna selection significantly improves backhaul and data transmission, making it an attractive solution in practical systems.
\end{abstract}

Index Terms-Small cell networks, full duplex, self-backhauling, antenna selection, inter-cell interference.

\section{INTRODUCTION}

Massive deployment of small cell networks provides a viable solution for future gigabit-level data communications [1]. When small cells are densely deployed in cellular networks, it is crucial to efficiently deliver backhaul data. Both wired and wireless links may be used for backhaul data transmissions. If data and backhaul share the radio spectrum, the overall system efficiency can be significantly improved [2], [3].

Recent studies show that the using of full duplex (FD) technique can not only to improve physical layer secrecy [4], but also to improve the backhaul and data transmission efficiency in small cell networks [5]. The FD transmission has attracted much attention as a viable approach in 5G networks to avoid the spectral efficiency loss with traditional half duplex (HD) transmission. While FD communications usually suffer from self-interference, i.e. the interference from its own transmission [6], recently developed antenna, RF and digital baseband technologies reduce the self-interference (SI) to noise level [6], [7]. In [8], the capacity performance of the FD selfbackhauling in a single small cell was analyzed. While the results in [8] demonstrate great potential in applying FD at small cell base stations, the system therein only considered one small cell and the analysis was based on the assumption that all interfering channels were approximated by their statistical averages. In practice, there are usually multiple small cells and interfering channels are timevarying. This motivates us to investigate the self-backhauling in small cell networks under more practical scenarios.

In this paper, we consider a multiple small cell network where both small cell base stations and mobile users are subject to interferences from neighboring small cell base stations. Further considering that small cell base stations are usually equipped with multiple antennas, we propose a novel dual antenna selection scheme at the small cell base stations. Antenna selection provides an efficient way to harvest the diversity gain by selecting a single antenna among a group of antennas. It also reduces the transceiver

G. Chen is with the Department of Engineering Science, University of Oxford, Oxford, UK, Emails: gaojie.chen@eng.ox.ac.uk.

Y. Gong is with the Advanced Signal Processing Group, School of Electronic, Electrical and Systems Engineering, Loughborough University, Loughborough, Leicestershire, UK, Email: y.gong@lboro.ac.uk.

P. Xiao and R. Tafazolli are with the Institute for Communication Systems, University of Surrey, Guildford, Surrey, UK, Emails: $\{p \cdot x i a o$ and r.tafazolli\}esurrey.ac.uk. complexity since only one RF chain is required at the transmitter and the receiver [9]. In the proposed scheme, the small cell base station is equipped with two groups of antennas, for receive and transmit antenna selection respectively. With dual antenna selection at the small cell base station, the performance of both backhauling and data transmission can be significantly improved. The main contributions of this paper are summarized as follows:

- Proposing a novel dual antenna selection scheme for the selfbackhauling in multiple small cell networks. Both the HD and FD at the small cell base stations are considered. To the best of authors' knowledge, this is the first time that the dual antenna selection approach is applied to multiple small cell networks. The proposed dual antenna selection provides an efficient way to transmit backhaul and data in small cell networks.

- Deriving closed-form expressions for the outage probabilities of the self-backhauling for both downlink and uplink in multismall cell networks. Unlike the analysis in [8] where interfering channels are approximated by their statistical averages, the outage analysis in this paper is based on more realistic assumptions that both the backhaul interference and residual self-interference (RSI) are modeled as Rayleigh fading. Furthermore interferences from neighboring base stations are also considered. Thus the analysis in this paper better reflects the practical scenario and shows deeper insights into the selfbackhauling system in small cell networks.

\section{Dual antennal Selection at TSBS}

A macro/small cell network with centralized backhaul approach [2] is shown in Fig. 1, where there are one macrocell base station $(M B S)$, one target small cell (TSC) and $N$ neighboring small cells $(N S C)$ with neighboring small base station $(N S B S)$. The base station in $T S C$ is the target-SBS (TSBS), and we assume there is one operating mobile user $(T U)$ in $T S C^{1}$. TSBS is connected to $M B S$ through a wireless backhaul link. The data received at the $T S B S$ and $T U$ are interfered by inter-cell interference (ICI) from neighboring $N S B S_{j}(j=1, \cdots, N)$.

In this paper, we propose to apply dual antenna selection at the $T S B S$. To be specific, we assume $T S B S$ is equipped with two groups of antennas, consisting of $K$ and $M$ antennas for receive and transmit antenna selection respectively. All other nodes are equipped with a single antenna ${ }^{2}$. For later use, the $k$-th receiving and $i$-th transmission antenna at TSBS are denoted as $T S B S_{k}(k=1, \cdots, K)$ and $T S B S_{i}(i=1, \cdots, M)$ respectively. The channel coefficients for $M B S \rightarrow T S B S_{k}, M B S \rightarrow T U$, $N S B S_{j} \rightarrow T S B S_{k}, N S B S_{j} \rightarrow T U$ and $T S B S_{i} \rightarrow T U$ are denoted as $h_{M S_{k}}, h_{M U}, h_{B_{j} S_{k}}, h_{B_{j} U}$ and $h_{S_{i} U}$ respectively. We assume all channels are independent Rayleigh flat fading, and keep unchanged within one packet but vary from one packet

${ }^{1}$ If there are several users in the target cell, only one user is operated through user scheduling [10] (e.g. random user selection).

${ }^{2}$ Although these nodes may also have multiple antennas, this will not affect the antenna selection schemes at $T S B S$. 
to another. The corresponding channel gains $\gamma_{j}=\left|h_{j}\right|^{2}$ are independently exponentially distributed with mean of $\lambda_{j}(j \in$ $\left.\left\{M S_{k}, M U, B_{j} S_{k}, B_{j} U, S_{i} U\right\}\right)$ respectively.

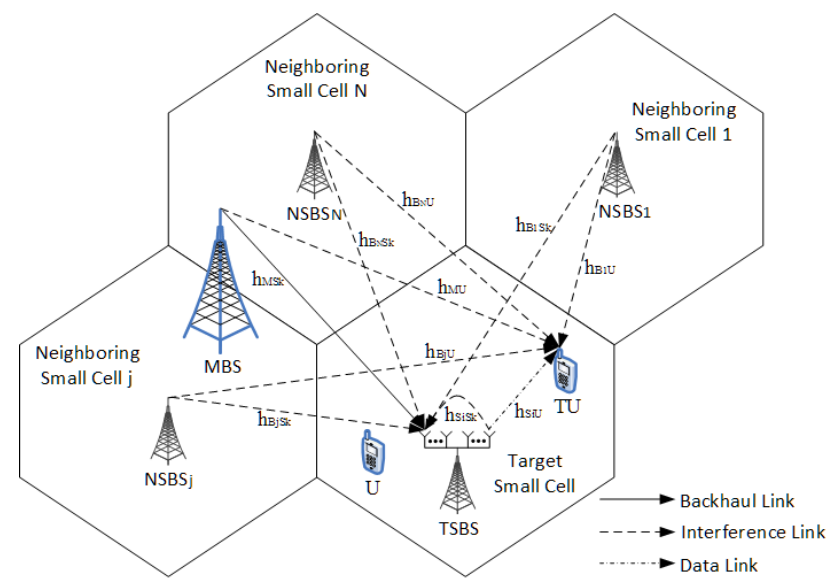

Fig. 1. A macro/small cell network with centralized backhaul approach.

Here we only consider the antenna selection for the downlink transmission. The uplink case can be similarly analyzed. $T S B S$ may operate either in half-duplex (HD) or full-duplex (FD) mode. With FD mode, $T S B S$ receives the backhaul data from $M B S$ and transmits its own data to $T U$ simultaneously. If the $k$-th receive and $i$-th transmit antennas are selected, the received signal at $T S B S$ is given by

$$
y_{B_{k}}=\sqrt{P_{M}} h_{M S_{k}} x_{M}+\sum_{j=1}^{N} \sqrt{P_{S}} h_{B_{j} S_{k}} x_{B_{j}}+\sqrt{P_{S}} h_{S_{i} S_{k}} x_{S}+n_{S_{k}},
$$

where the first term corresponds to the backhaul data $x_{M}$ from $M B S$, the second term contains ICI $x_{B_{j}}$ from neighboring $S B S$ $\mathrm{s}$, the third term is the residual self-interference $x_{S}$ from the $j$ th transmission antenna to the $k$-th receiving antenna, $n_{S_{k}}$ is the additive white Gaussian noise (AWGN), and $P_{M}$ and $P_{S}$ are the transmission powers at $M B S$ and $S B S$ respectively. When the self-interference is significantly suppressed, $h_{S_{i} S_{k}}$ can be regarded as an independent Rayleigh distributed variable (e.g. [11]). Because $T S B S$ operates in the FD mode, it simultaneously transmits $x_{S}$ to $T U$ while receiving $x_{M}$. In a general case when $T U$ also receives $x_{M}$ from $M B S$, the received data signal at $T U$ is given by

$$
y_{U_{i}}=\sqrt{P_{S}} h_{S_{i} U} x_{S}+\sum_{j=1}^{N} \sqrt{P_{S}} h_{B_{j} U} x_{B_{j}}+\sqrt{P_{M}} h_{M U} x_{M}+n_{U} .
$$

Without loss of generality, we assume that all noise variances and transmission powers are normalized to unity. In this paper, we consider interference limited scenario that the performance is dominated by interferences and the influence from noise is trivial. From (1) and (2), the instantaneous receiving signal-to-interference ratio (SIR) at $T S B S$ and $T U$ can be obtained as

$$
\gamma_{B_{k}}=\frac{\gamma_{M S_{k}}}{\sum_{j=1}^{N} \gamma_{B_{j} S_{k}}+\gamma_{S_{i} S_{k}}} \text { and } \gamma_{U_{i}}=\frac{\gamma_{S_{i} U}}{\sum_{j=1}^{N} \gamma_{B_{j} U}+\gamma_{M U}} \text {, }
$$

respectively. The data at $T U$ are either for locally use in the same small cell, or for the core network via MBS. In the latter case, the system is equivalent to a relay network and the SIR for the overall system is given by [8]

$$
\gamma_{H}=\min \left(\gamma_{B_{k}}, \gamma_{U_{i}}\right) .
$$

This paper analyzes the system throughput based on (4). If the data are local, throughput for access and backhaul links are separately obtained, but the derivation is not shown in this paper due to the space constraint ${ }^{3}$.

The optimum transmission and receiving antennas at $T S B S$ should be selected by maximizing (4). It is clear from (3) that $\gamma_{B_{k}}$ depends on both receiving and transmission antennas. Thus the optimum transmission and receiving antennas must be jointly selected by comparing (4) for all possible receiving/transmission pairs. This involves extensive computation. Next we present a suboptimum solution to 'de-couple' the transmission and receiving antenna selection. Noticing from (3) that $\gamma_{U_{i}}$ only depends on the transmission antenna, we can firstly select the transmission antenna $\left(S_{t}\right)$ by maximizing $\gamma_{U_{i}}$ as

$$
S_{t}=\arg \max _{i \in(1 \ldots M)} \frac{\gamma_{S_{i} U}}{\sum_{j=1}^{N} \gamma_{B_{j} U}+\gamma_{M U}},
$$

With the selected transmission antenna $S_{t}$, the receiving antenna $\left(S_{r}\right)$ is then selected by maximizing $\gamma_{B_{k}}$ as

$$
S_{r}=\arg \max _{k \in(1 \ldots K)}\left\{\frac{\gamma_{M S_{k}}}{\sum_{j=1}^{N} \gamma_{B_{j} S_{k}}+\gamma_{S_{t} S_{k}}}\right\}
$$

The sub-optimum selection scheme has very close performance to the optimum scheme. This follows from the fact that, as is shown in (3), the transmission antenna affects the receive antenna selection through the residual self-interference term $\gamma_{S_{i} S_{k}}$. If selfinterference is significantly suppressed, $\gamma_{S_{i} S_{k}}$ will have little effect on receive antenna selection. If $T S B S$ operates in the HD mode where it receives and transmits data at different time slots, the received signals at the $T S B S$ and $T U$ are free from the selfinterference and interference from $M B S$ respectively. The transmit and receive antenna selection can be similarly obtained, and the 'sub-optimum' scheme described above becomes optimum (because no self-interference is involved).

\section{Average throughrut}

First, we consider the FD TSBS antenna selection. For delaylimited transmission, the average throughput is obtained as

$$
T^{F D}=R\left(1-P_{\text {out }}^{F D}\left(\gamma_{t h}\right)\right),
$$

where $R(\mathrm{bps} / \mathrm{Hz})$ is the transmission rate, $P_{\text {out }}^{F D}\left(\gamma_{t h}\right)=P\left(\gamma_{H}<\right.$ $\left.\gamma_{t h}\right)$ is the outage probability with $\gamma_{t h}=2^{R}-1$, and $P($.) denotes the probability of the enclosed. We have the following proposition.

Proposition 1: If the TSBS operates in the FD mode, the proposed antenna selection in downlink transmission has the outage probability as (8) at the top of next page, where $\mathcal{F}_{2,1}(a, b, c, z)$ is the first hypergeometric function, and $C_{M-1}^{l}=M-1 ! /[l !(M-$ $1-l)$ !] which is the binomial coefficient, $\alpha=\frac{\lambda_{S_{i} S_{k}}-\lambda_{B_{j} S_{k}}}{\lambda_{S_{i} S_{k} \lambda_{B_{j} S_{k}} \gamma_{t h}}}$, $\beta=\frac{\lambda_{M S}+\lambda_{S_{i} S_{k}} \gamma_{t h}}{\lambda_{M S} \lambda_{S_{i} S_{k}} \gamma_{t h}}, \alpha_{1}=\frac{\lambda_{B_{j} S_{k}} \gamma_{t h}}{\lambda_{B_{j} S_{k}} \gamma_{t h}+\lambda_{M S}}, \beta_{1}=\frac{\lambda_{M S} \lambda_{B_{j} S_{k}} \gamma_{t h}}{\lambda_{M S}+\lambda_{B_{j} S_{k}} \gamma_{t h}}$, $\alpha_{2}=\frac{\lambda_{M U}-\lambda_{B_{j} U}}{\lambda_{M U} \lambda_{B_{j} U} \gamma_{t h}}$ and $\beta_{2}=\frac{\gamma_{t h}(l+1) \lambda_{M U}+\lambda_{S_{i} U}}{\gamma_{t h} \lambda_{M U} \lambda_{S_{i} U}}$.

\section{Proof: See Appendix I.}

It is interesting to note that when the ICI from $N S B S$-s is ignored (e.g. [2]), (8) can be simplified as

$$
\begin{aligned}
P_{\text {out }}^{F D}\left(\gamma_{t h}\right)= & -\left[1-\left(\frac{\gamma_{t h} \lambda_{S_{i} S_{k}}}{\gamma_{t h} \lambda_{S_{i} S_{k}}+\lambda_{M S}}\right)^{K}\right] \\
& \cdot\left[1-\sum_{l=0}^{M} C_{M}^{l}(-1)^{l} \frac{\lambda_{S_{i} U} e^{-\frac{\gamma_{t h} l}{\lambda_{S_{i} U}}}}{\gamma_{t h} l \lambda_{M U}+\lambda_{S_{i} U}}\right] .
\end{aligned}
$$

${ }^{3}$ The distributions for the access and backhaul SIR are obtained as (15) and (16) respectively, and substituting them into (7) immediately gives the corresponding throughput. 


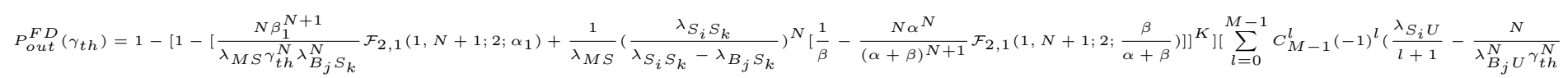

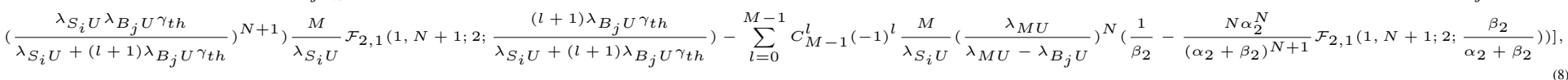

On the other hand, in the HD mode, because the $T S B S$ does not receive and transmit simultaneously, the average throughput is obtained as

$$
T^{H D}=\frac{1}{2} R\left(1-P_{\text {out }}^{H D}\left(\gamma_{t h}\right)\right) .
$$

Following the similar procedures to those for the FD mode, the outage probability for the HD mode can be obtained as

$$
\begin{aligned}
& P_{\text {out }}^{H D}\left(\gamma_{t h}\right)=1-\left[1-\left(\frac{\lambda_{M S}}{\gamma_{t h} \lambda_{B_{j} S_{k}}+\lambda_{M S}}\right)^{N}\right]^{K} \\
& \cdot\left(1-\sum_{l=0}^{M} C_{M}^{l}(-1)^{l}\left[\frac{\lambda_{S_{i} U}}{\gamma_{t h} l \lambda_{B_{j} U}+\lambda_{S_{i} U}}\right]^{N}\right) .
\end{aligned}
$$

Particularly, if the ICI from $N S B S$-s is ignored, (11) becomes

$$
P_{\text {out }}^{H D}\left(\gamma_{t h}\right)=1-\left[1-\left(1-e^{\frac{\gamma_{t h}}{\lambda_{M S}}}\right)^{K}\right]\left[1-\left(1-e^{\frac{\gamma_{t h}}{\lambda_{S_{i} U}}}\right)^{M}\right] \text {. }
$$

\section{NumERicAl Simulations}

In the simulations, all noise variance and transmission powers are normalized to unity. For comparison, the results for the traditional scheme that no antenna selection is applied at the target small base station are also shown. Both theoretical and simulation results are shown, where simulation results are obtained by averaging $10^{6}$ independent Monte Carlo runs. In all cases, the simulation results well match the analysis, which well verifies the closed-form outage probabilities derived in this paper.

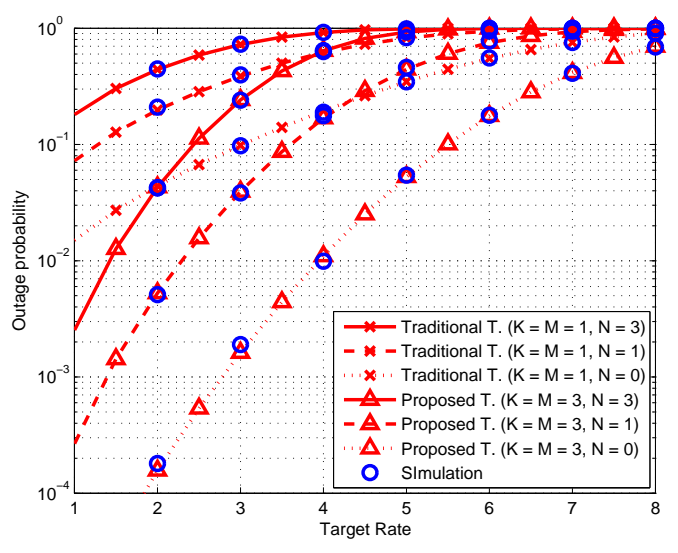

Fig. 2. The outage probabilities $v s$ target rate in the FD modes.

Fig. 2 compares the outage probabilities in the FD mode for the proposed and traditional schemes, where the average channel gains are set as $\lambda_{M S}=\lambda_{S_{i} U}=30 \mathrm{~dB}, \lambda_{B_{j} S_{k}}=\lambda_{B_{j} U}=15$ $\mathrm{dB}, \lambda_{S_{i} S_{k}}=5 \mathrm{~dB}$ and $\lambda_{M U}=10 \mathrm{~dB}$. It is clearly shown that the proposed scheme has significant better outage performance than the traditional scheme (without antenna selection). Particularly in the proposed scheme, the outage probability decreases consistently with more antennas. It is also evident that, with more inter-cell interference (i.e. larger $N$ ), the outage probability increases significantly. Fig. 3 compares the average throughputs in the FD mode with respective to the channel SNR, where the average channel gains are set as $\lambda_{B_{j} S_{k}}=\lambda_{B_{j} U}=10 \mathrm{~dB}, \lambda_{S_{i} S_{k}}=5 \mathrm{~dB}, \lambda_{M U}=10$

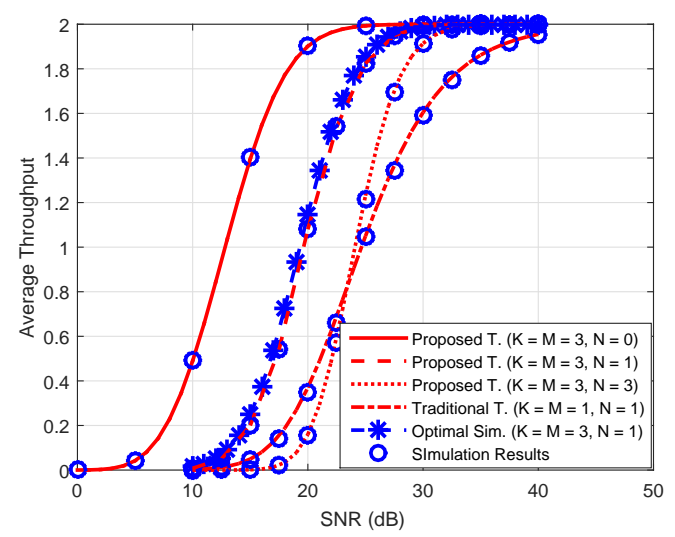

Fig. 3. The average throughput $v s$ channel SNR in the FD mode.

$\mathrm{dB}$ and $R_{T}=2 \mathrm{~b} / \mathrm{s} / \mathrm{Hz}$. It is clearly shown that the average throughput of proposed suboptimal scheme is consistently higher than that of the traditional scheme. For comparison, Fig. 3 also shows the result for the optimum scheme that the optimum antennas are selected by comparing (4) for all possible receiving/transmission pairs. As was expected, the proposed suboptimum scheme has similar performance as the optimum scheme.

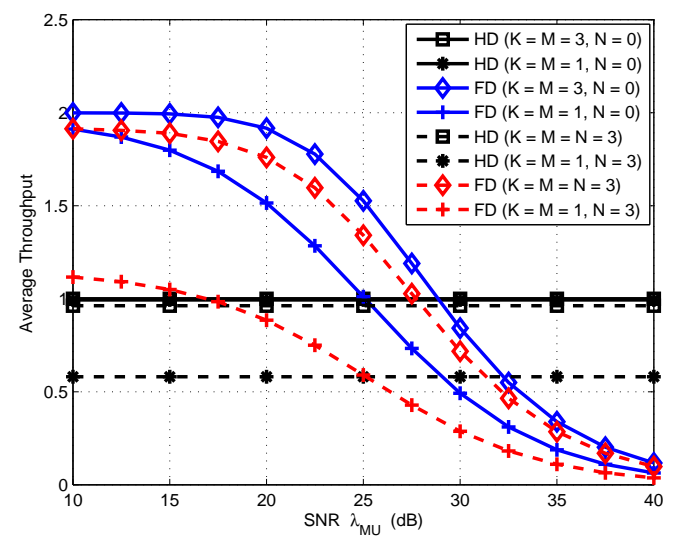

Fig. 4. Average throughput $v s$ different $\lambda_{M U}$ in FD and HD modes.

Fig. 4 compares the average throughput between the HD and FD modes with respective to different $\lambda_{M U}$, where $\lambda_{M S}=\lambda_{S_{i} U}=30$ $\mathrm{dB}, \lambda_{B_{j} S_{k}}=\lambda_{B_{j} U}=15 \mathrm{~dB}, \lambda_{S_{i} S_{k}}=5$ and $R_{T}=2 \mathrm{~b} / \mathrm{s} / \mathrm{Hz}$. It is clearly shown that, for different $\lambda_{M U}$, the throughput in the FD mode can be either higher or lower than that in the HD mode. For example, for the cases of $K=M=3$ and $K=M=1$, when $N=3$ (i.e. in the presence of intercell interference), the average throughput for FD is higher than that for HD when $\lambda_{M U}$ is approximately smaller than $28.5 \mathrm{~dB}$, and otherwise the HD mode has higher throughput. Fig. 5 compares average throughputs between the HD and FD modes with respect to different $\lambda_{S_{i} S_{k}}$, where $\lambda_{M S}=\lambda_{S_{i} U}=30 \mathrm{~dB}, \lambda_{B_{j} S_{k}}=\lambda_{B_{j} U}=15 \mathrm{~dB}, \lambda_{M U}=10$ and $R_{T}=2 \mathrm{~b} / \mathrm{s} / \mathrm{Hz}$. Similar to the results shown in Fig. 4, for different $\lambda_{S_{i} S_{k}}$, the FD may or may not have higher average throughputs than the HD mode. Fig. 4 and 5 imply that, according to different channel conditions, we can switch between HD and FD 


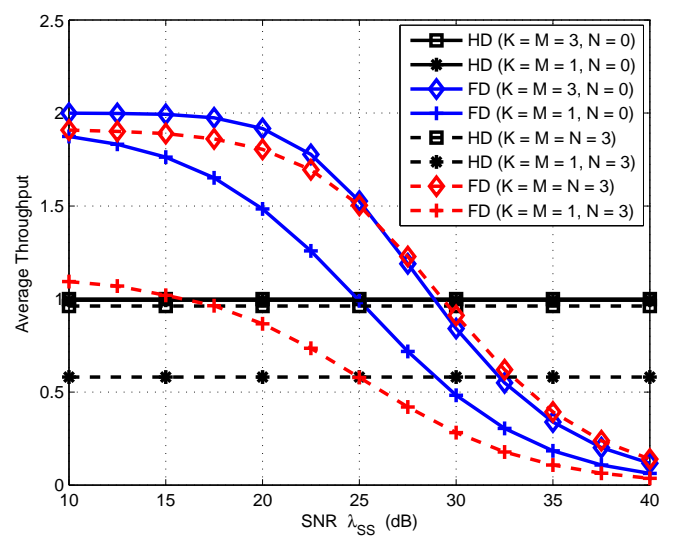

Fig. 5. Average throughput $v s$ different $\lambda_{S_{i} S_{k}}$ in FD and HD modes.

mode for higher throughput.

\section{CONCLUSiOnS}

This paper proposed a dual antenna selection strategy in multiple small cell networks, where both HD and FD modes at $T S B S$ were considered. Analysis showed that the FD mode at TSBS may or may not lead to higher average throughput than the HD mode. Switching between half and duplex transmission at TSBS may thus be necessary, which will be left as a future topic of research. In all cases, the dual antenna selection at $T S B S$ mitigates the ICI from neighboring $S B S$-s, and significantly improves the backhaul and data transmission, making it an attractive solution to realize efficient backhaul transmission in multiple small cell networks.

\section{APPENDIX I - PROOF OF (8)}

We define $x=\gamma_{M S_{k}}, y=\sum_{j=1}^{N} \gamma_{B_{j} S_{k}}, x_{1}=\max _{i \in(1 \ldots M)}\left\{\gamma_{S_{i} U}\right\}$, $y_{1}=\sum_{j=1}^{N} \gamma_{S_{i} U}, y_{2}=\sum_{j=1}^{N} \gamma_{B_{j} S_{k}}+\gamma_{S_{i} S_{k}}$ and $y_{3}=$ $\sum_{j=1}^{N} \gamma_{B_{j} U}+\gamma_{M U}$. The CDF of $x$ and $x_{1}$ can be obtained as $F_{X}(x)=1-e^{-\frac{x}{\lambda_{M S}}}$ and $F_{X_{1}}\left(x_{1}\right)=\left(1-e^{-\frac{x_{1}}{\lambda_{S_{i} U}}}\right)^{M}=$ $\sum_{l=0}^{M} C_{M}^{l}(-1)^{l} e^{-\frac{l x_{1}}{\lambda S_{i} U}}$, respectively. The PDF of $y$ and $y_{1}$ are given by $f_{Y}(y)=\frac{y^{N-1} e^{-\frac{y}{\lambda_{B_{j} S_{k}}}}}{\lambda_{B_{j} S_{k}} \Gamma(N)}$ and $f_{Y_{1}}\left(y_{1}\right)=\frac{y_{1}^{N-1} e^{-\frac{y_{1}}{\lambda_{B_{j} U}}}}{\lambda_{B_{j} U}^{N} \Gamma(N)}$ respectively, where $\Gamma$ (.) denotes the gamma function. And the CDF of $y_{2}$ and $y_{3}$ can be obtained as

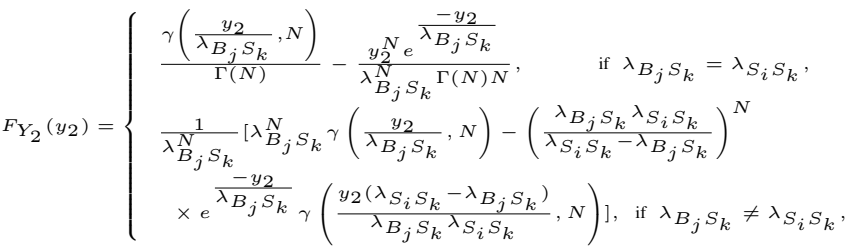

$$
\begin{aligned}
& F_{Y_{3}}\left(y_{3}\right)=\left\{\begin{array}{l}
\frac{\gamma\left(\frac{y_{3}}{\lambda_{B_{j} U} U}, N\right)}{\Gamma(N)}-\frac{y_{3}^{N} e^{\frac{-y_{3}}{\lambda_{B_{j}} U}}}{\lambda_{B_{j}}^{N} U^{\Gamma(N) N}}, \quad \text { if } \lambda_{B_{j} U}=\lambda_{M U}, \\
\frac{1}{\lambda_{B_{j} U}^{N}}\left[\lambda_{B_{j} U}^{N} \gamma\left(\frac{y_{2}}{\lambda_{B_{j} U}}, N\right)-\left(\frac{\lambda_{B_{j} U \lambda_{M U}}}{\lambda_{M U}-\lambda_{B_{j} U}}\right)^{N}\right. \\
\left.\quad \times e^{\frac{-y_{3}}{\lambda_{B_{j} U}}} \gamma\left(\frac{y_{3}\left(\lambda_{M U}-\lambda_{B_{j} U}\right)}{\lambda_{B_{j} U^{\lambda} M U}}, N\right)\right], \text { if } \lambda_{B_{j} U} \neq \lambda_{M U},
\end{array}\right.
\end{aligned}
$$

where $\gamma(a, b)$ denotes the lower incomplete gamma function. Next, let $w_{2}=\max _{k \in(1 \ldots K)}\left(x / y_{2}\right)$, the CDF of $w_{2}$ can be obtained

$$
\begin{aligned}
& F_{W_{2}}\left(w_{2}\right)=\left[\int_{0}^{\infty} f_{X}(x) F_{Y_{2}}\left(w_{2} / x\right) d x\right]^{K}= \\
& \left\{\begin{array}{l}
{\left[1-\left(\frac{\lambda_{M S}}{w_{2} \lambda_{B_{j} S}{ }^{\lambda} M S}\right)^{N+1}\right]^{K}, \quad \text { if } \lambda_{B_{j} S_{k}}=\lambda_{S_{i} S_{k}},} \\
\left(\frac{N \beta_{1}^{N+1}}{\lambda_{M S} \gamma_{t h}^{N} \lambda_{B j}^{N} S_{k}} \mathcal{F}_{2,1}\left(1, N+1 ; 2 ; \alpha_{1}\right)+\frac{1}{\lambda_{M S}}\left(\frac{\lambda_{S_{i} S_{k}}}{\lambda_{S_{i} S_{k}-\lambda_{B} S_{k}}}\right)^{N}\left(\frac{1}{\beta}\right.\right. \\
\left.\left.-\frac{N \alpha{ }^{N}}{(\alpha+\beta)^{N+1}} \mathcal{F}_{2,1}\left(1, N+1 ; 2 ; \frac{\beta}{\alpha+\beta}\right)\right)\right)^{K}, \text { if } \lambda_{B_{j} S_{k}} \neq \lambda_{S_{i} S_{k}}
\end{array}\right.
\end{aligned}
$$

where $\mathcal{F}_{2,1}(a, b, c, z)$ is the first hypergeometric function, $\alpha=$ $\frac{\lambda_{S_{i} S_{k}}-\lambda_{B_{j} S_{k}}}{\lambda_{S_{i} S_{k} \lambda_{B_{j} S_{k}} \gamma_{t h}}}, \beta=\frac{\lambda_{M S}+\lambda_{S_{i} S_{k}} \gamma_{t h}}{\lambda_{M S} \lambda_{S_{i} S_{k}} \gamma_{t h}}, \alpha_{1}=\frac{\lambda_{B_{j} S_{k}} \gamma_{t h}}{\lambda_{B_{j} S_{k}} \gamma_{t h}+\lambda_{M S}}$ and $\beta_{1}=\frac{\lambda_{M S} \lambda_{B_{j} S_{k}} \gamma_{t h}}{\lambda_{M S}+\lambda_{B_{j} S_{k}} \gamma_{t h}}$. Then, we let $w_{3}=x_{1} / y_{3}$ and the PDF of $x_{1}$ can be obtained as $f_{X_{1}}\left(x_{1}\right)=\frac{M}{\lambda_{S_{i} U}} \sum_{l=0}^{M-1} C_{M-1}^{l}(-1)^{l} e^{-\frac{(l+1) x_{1}}{\lambda_{S_{i}} U}}$, then, the $\mathrm{CDF}$ of $w_{3}$ can be obtained as

$$
\begin{aligned}
& F_{W_{3}}\left(w_{3}\right)=\int_{0}^{\infty}{ }_{f_{X_{1}}\left(x_{1}\right) F_{Y_{3}}\left(w_{3} / x_{1}\right) d x_{1}=} \\
& \left\{\begin{array}{cc}
\sum_{l=0}^{M} C_{M}^{l}(-1)^{l}\left(\frac{\lambda_{S_{i} U}}{w_{1} l \lambda_{B_{j} U^{\lambda} S_{i} U}}\right)^{N+1}, \quad \text { if } \lambda_{B_{j} U}=\lambda_{M U}, \\
1-\sum_{l=0}^{M-1} C_{M-1}^{l}(-1)^{l} \frac{M}{\lambda_{S_{i} U}}\left(\frac{\lambda_{S_{i} U}}{l+1}-\frac{N}{\lambda_{B_{j} U^{N}}^{N} \gamma_{t h}^{N}}\left(\alpha_{3} \lambda_{S_{i} U}\right)^{N+1}\right) \\
\times \mathcal{F}_{2,1}\left(1, N+1 ; 2 ; \alpha_{3}(l+1)\right)+\sum_{l=0}^{M-1} C_{M-1}^{l}(-1)^{l} \frac{M}{\lambda_{S_{i} U}} \\
\times\left(\frac{\lambda_{M U}}{\lambda_{M U}-\lambda_{B_{j} U}}\right)^{N}\left(\frac{1}{\beta_{2}}-\frac{N \alpha_{2}^{N}}{\left(\alpha_{2}+\beta_{2}\right)^{N+1}} \mathcal{F}_{2,1}\left(1, N+1 ; 2 ; \frac{\beta_{2}}{\alpha_{2}+\beta_{2}}\right)\right), \\
\text { if } \lambda_{B_{j} U} \neq \lambda_{M U},
\end{array}\right.
\end{aligned}
$$

where $\alpha_{2}=\frac{\lambda_{M U}-\lambda_{B_{j} U}}{\lambda_{M U} \lambda_{B_{j}} \gamma_{t h}}, \beta_{2}=\frac{\gamma_{t h}(l+1) \lambda_{M U}+\lambda_{S_{i} U}}{\gamma_{t h} \lambda_{M U} \lambda_{S_{i} U}}$ and $\alpha_{3}=$ $\frac{\lambda_{B_{j} U} \gamma_{t h}}{\lambda+(l+1) \lambda_{B_{j} U} \gamma_{t h}}$. Finally, let $z_{1}=\min \left(w_{2}, w_{3}\right)$, and use (15) and (16), the CDF of $z_{1}$ can be obtained by $F_{Z_{1}}\left(z_{1}\right)=1-(1-$ $\left.F_{W_{2}}\left(w_{2}\right)\right)\left(1-F_{w_{3}}\left(w_{3}\right)\right)$.

\section{REFERENCES}

[1] E. Hossain, M. Rasti, H. Tabassum, and A. Abdelnasser, "Evolution toward 5G multi-tier cellular wireless networks: An interference management perspective," IEEE Wireless Commun., vol. 21, no. 3, pp. 118-127, June 2014.

[2] X. Ge, H. Cheng, M. Guizani, and T. Han, " $5 \mathrm{G}$ wireless backhaul networks: Challenges and research advances," IEEE Network, vol. 28, no. 6, pp. 6-11, Nov. 2014.

[3] R. A. Pitaval, O. Tirkkonen, R. Wichman, K. Pajukoski, E. Lahetkangas, and E. Tiirola, "Full-duplex self-backhauling for small-cell 5G networks," IEEE Wireless Commun., vol. 22, no. 3, pp. 83-89, Oct. 2015.

[4] G. Chen, Y. Gong, P. Xiao, and J. A. Chambers, "Physical layer network security in the full-duplex relay system," IEEE Trans. Inform. Forensics and Security, vol. 10, no. 3, pp. 574-583, Apr. 2015.

[5] S. Hong, J. Brand, J. Choi, M. Jain, J. Mehlman, S. Katti, and P. Levis, "Applications of self-interference cancellation in 5G and beyond," IEEE Commun. Mag., vol. 52, no. 2, pp. 114-121, Feb. 2014.

[6] B. Debaillie, D. J. Broek, C. Lavin, B. Liempd, E. A. M. Klumperink, C. Palacios, J. Craninckx, B. Nauta, and A. Parssinen, "Analog/RF solutions enabling compact full-duplex radios," IEEE J. Sel. Areas Commun., vol. 32, no. 9, pp. 1662-1673, Oct. 2014.

[7] J. I. Choi, M. Jain, K. Srinivasan, P. Levis, and S. Katti, "Achieving single channel, full duplex wireless communication," MobiCom, Chicago, USA, Sep. 2010.

[8] U. Siddique, H. Tabassum, and E. Hossain, "Adaptive in-band self-backhauling for full-duplex small cells," in Proc. ICC, London, UK, June 2015.

[9] S. Sanayei and A. Nosratinia, "Antenna selection in MIMO systems," IEEE Commun. Mag., vol. 42, no. 10, pp. 68-73, Oct. 2004.

[10] G. Chen, Y. Gong, and J. A. Chambers, "Study of relay selection in a multi-cell cognitive network," IEEE Wireless Commun. Lett., vol. 11, no. 6, pp. 23512354, June 2012.

[11] I. Krikidis, H. A. Suraweera, P. J. Smith, and C. Yuen, "Full-duplex relay selection for amplify-and-forward cooperative networks," IEEE Trans. Wireless Commun., vol. 11, no. 12, pp. 4381-4393, Dec. 2012. 